\title{
Effects of Asymmetries on the Dynamics of Motorized Momentum Exchange Tether and Payloads Injection Precision
}

\author{
Naiming Qi, ${ }^{1}$ Yong Yang, ${ }^{1}$ Jun Zhao, ${ }^{1}$ Qilong Sun, ${ }^{1}$ and Wenhui Zhang ${ }^{2}$ \\ ${ }^{1}$ School of Astronautics, Harbin Institute of Technology, Harbin 150001, China \\ ${ }^{2}$ Institute of Engineering and Design, Lishui University, Lishui 323000, China \\ Correspondence should be addressed to Yong Yang; yangyong_hit@hit.edu.cn
}

Received 2 September 2015; Revised 30 November 2015; Accepted 7 December 2015

Academic Editor: Hyochoong Bang

Copyright ( 2015 Naiming Qi et al. This is an open access article distributed under the Creative Commons Attribution License, which permits unrestricted use, distribution, and reproduction in any medium, provided the original work is properly cited.

\begin{abstract}
This paper presents the error dynamic model of motorized momentum exchange tether (MMET) based on the momentum exchange principle of space tether. The error dynamics are caused by the structural bias of the differences in tethers' length and the difference in payloads' mass. After that, the coupling analysis between orbit and attitude is presented. It is shown that, with increasing the differences in tethers' length and payloads' mass, the COM deviation of the MMET increases linearly. The numerical simulations of the MMET by considering the structural asymmetries are presented; the results show that the asymmetries have tiny influences on the orbit of the chief satellite by decreasing the apogee, which will change the instantaneous velocity at the apogee and affect the payload injection precision. What is more, the structural asymmetries have effects on the attitude elements (including the pitch angle and yaw angle); however, the effects could be weakened by the external torque. The structural asymmetries and gravity gradient torque have composite effects on the angular velocity of the propulsion tether.
\end{abstract}

\section{Introduction}

With the development of exploiting the interplanetary space, human beings will establish the permanent colonies in outer space, such as the moon or planets like Mars. As a result, one of the issues of transferring massive materials (food, water, oxygen, etc.) to those planets and the resources from these locations back to Earth has to be solved. However, the disadvantages of traditional rocket systems, for instance, the low transfer efficiency, high cost, and nonreusability, are excessive. These missions will require innovative, propulsionsaving approaches and reusable vehicles. A space tether based on the momentum exchange principle and electrodynamic force has many unique advantages and is the subject of this paper.

In 1985, Joseph et al. illustrated the possible applications of a space tether, such as electrodynamic tether to reboost orbit or remove debris [1-4], especially the application of a momentum exchange tether for transferring payloads to other planets. In 1980, Robert proposed a new concept of momentum exchange/electrodynamic-reboost tether
(MXER) for the first time [5-7]. For improving the payload transference efficiency, a new kind of applications of the space tether, denoted as motorized momentum exchange tether (MMET), was proposed and has been a hot research topic over the last two decades [8] and further investigated by Robert and Ziegler $[9,10]$. Additionally, by considering the effect of the tether's axial and torsional oscillation, Chen and Cartmell studied the dynamics of the MMET with axial and torsional oscillation using Lagrange methods and controlled the in-plane angle of the MMET by hybrid sliding mode control strategies [11, 12]. In 2013, Murray studied the moontracking orbit for the payload transfer periodically $[13,14]$. It was shown that if the ascending (or descending) node of the moon's orbit around Earth could be tracked effectively and periodically, the payload launched by the MMET could arrive at the nodes while the moon arrives at this area and rendezvoused with the moon successively.

Recently, Yang et al., studied the dynamics of the Continuous Cislunar Payloads Transfer System (CCPTS) with tapered tether [15]. It was illustrated that the energy of the CCPTS with tapered tether is lower than the energy of the CCPTS 
with uniform tether for the same preconditions. Therefore, the CCPTS with tapered tether could decrease the total mass and save energy without reducing the performance of the system. Furthermore, the two-dimensional error dynamics of the CCPTS, which considered the structural bias caused by tether lengths' deviation and payloads' mass deviation, were studied preliminarily [16]. The results showed that the asymmetries of the CCPTS had similar influence on CCPTS's dynamics; with increasing the tether length deviation or payload mass deviation, the maximum of the CCPTS's errors of generalized coordinates (as well as generalized velocities) increased linearly over time. However, they did not consider the out-of-plane movement of the propulsion tether and did not analyze the influence of the asymmetries on the payloads injection precision.

Though the MMET has many advantages such as saving energy and reusability, the key to transfer the payload between the Earth and the moon successfully is determined by the structural and mass symmetry of the MMET. Consequently, the main focus of this paper is to build the threedimensional (3D) rigid error dynamics of the MMET and study the influence of asymmetries on the dynamics of the MMET. The structure of the paper is as follows: in Section 1, a brief introduction to space tethers and MMET is presented firstly. In Section 2, the conception of the MMET and payload cislunar transfer are introduced. In Section 3, we list some reasonable assumptions and define some coordinates; in Section 4, we build the 3D rigid error dynamics model by Lagrange methods [17]; in Section 5, we present the orbit and attitude coupling analysis and deduce the expression of the external centrifugal force caused by the structure bias; in Section 6, we present the numerical simulations and investigate the effects of the asymmetries on the dynamics of the MMET dynamics; in Section 7, the theoretical analysis of the effect of asymmetries on the payload injection precision is presented. Conclusions are given in the last section.

\section{Conceptions of the MMET and Payload Transfer}

Figure 1 shows schematic of a MMET [10]. The MMET contains four main parts: (1) the chief satellite (contains the control system, stator, and rotor, etc.); (2) the outrigger system (for balance); (3) propulsion tether (provides required velocity increment for the payload); (4) two payloads (the one coming to the moon and the other going back to the Earth). When the propulsion tether is deployed completely, the outrigger system will be retrieved into the chief satellite. Figure 2 shows the schematic of the procedures of the MMET tossing the payload. In this figure, $\Delta v$ refers to the velocity increments supplied by the rotation of the propulsion tether, $v_{p}$ refers to the velocity of the chief satellite at perigee. We can observe that, in Step 1, a payload $P_{1}$ which needs to be transferred to the moon will be sent to the place below the perigee of the MMET's parking orbit, and the other payload $P_{2}$ from the moon reaches the area above the perigee of the MMET's parking orbit simultaneously. At the same time, the two end effectors attached at the end of the propulsion tethers

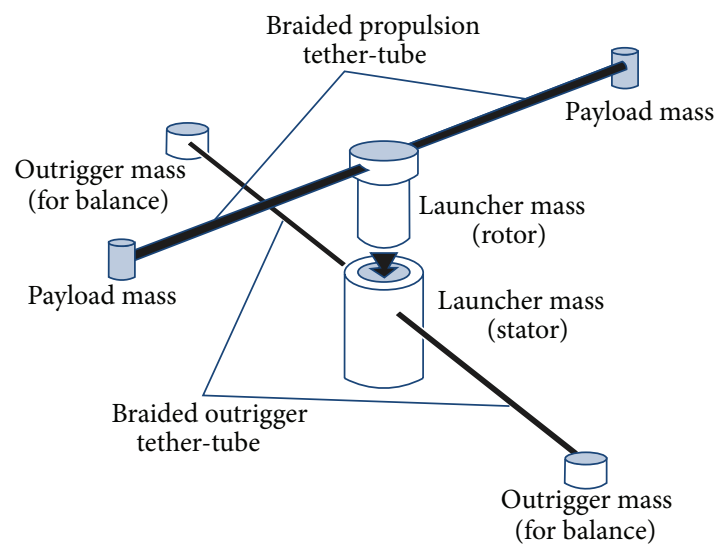

Figure 1: Schematic of a MMET and its components [9].

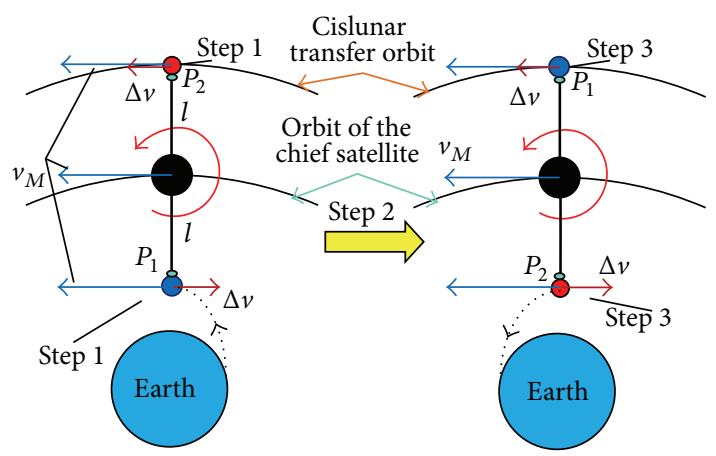

FIgURE 2: Schematic of a MMET transferring payloads.

catch the two payloads; Step 2 shows that, after capturing the two payloads, the orbit of the MMET requires to be modified, after orbiting Earth for some time, when the MMET reaches the perigee; the locations of $P_{1}$ and $P_{2}$ have been switched ( $P_{1}$ reaches the upper tip of the tether and $P_{2}$ reaches the lower tip); Step 3 shows that $P_{1}$ obtains the required velocity increment and is tossed into the cislunar transfer trajectory and $P_{2}$ is sent back to Earth.

\section{Coordinate Systems of the MMET}

In order to build the $3 \mathrm{D}$ rigid error dynamic model and motion equations of the MMET, we need to set relevant hypotheses as follows:

(1) The payloads on the tether tips have the same shape but different weight.

(2) The bending deformation and axial elasticity deformation of the propulsion tethers are ignored.

(3) The third-body gravitational force and the Earth oblateness and other perturbing forces are neglected.

Based on those assumptions above, some required coordinate systems are defined as follows:

(1) The geocentric equatorial inertial coordinate $O_{E} X Y Z$, shown in Figure 3: the origin of the coordinate is the 


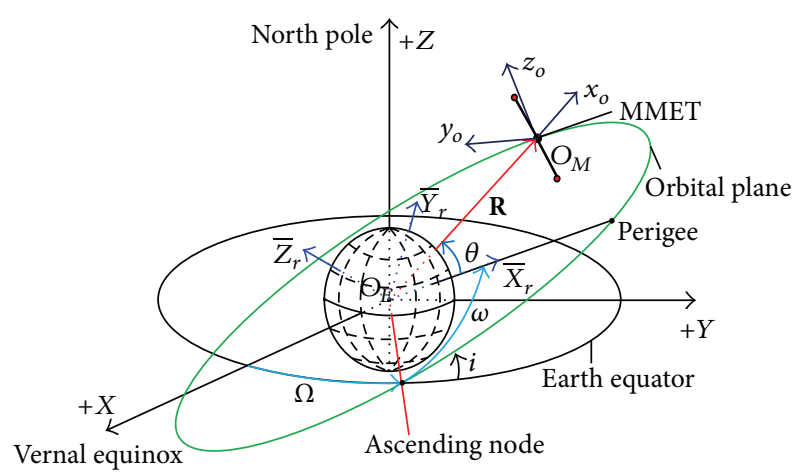

FIgURE 3: The coordinate systems.

Earth's COM, axis $O_{E} X$ points to the direction of vernal equinox in Earth equatorial plane, axis $O_{E} Y$ is perpendicular to axis $O_{E} X$ in Earth equatorial plane, and axis $O_{E} Z$ follows the right-handed rule about axis $O_{E} X$ and $O_{E} Y$; the unit vectors of each axis are presented as $\mathbf{I}, \mathbf{J}$, and $\mathbf{K}$.

(2) Orbital plane coordinate $O_{E} \bar{X}_{r} \bar{Y}_{r} \bar{Z}_{r}$ : the origin refers to the Earth's center, axis $O_{E} \bar{X}_{r}$ points to the perigee of the MMET, axis $O_{E} Y_{r}$ is perpendicular to the axis $O_{E} \bar{X}_{r}$ and lies in the orbital plane, and axis $O_{E} \bar{Z}_{r}$ follows the right-handed rule of the coordinate system; the unit vectors of each axis of $O_{E} \bar{X}_{r} \bar{Y}_{r} \bar{Z}_{r}$ are presented as $\mathbf{i}, \mathbf{j}$, and $\mathbf{k}$. Without considering the other perturbations, the orbital plane coordinate $O_{E} \bar{X}_{r} \bar{Y}_{r} \bar{Z}_{r}$ can be treated as an relative inertia coordinate.

(3) Orbit coordinate $O_{M} x_{o} y_{o} z_{o}$ : the origin is the centerof-mass (COM) of the chief satellite, axis $O_{M} x_{o}$ lies along the direction of MMET's radius vector, axis $O_{M} y_{o}$ is perpendicular to the axis $O_{M} x_{o}$ and lies in the orbital plane, and axis $O_{M} z_{o}$ follows the right-handed rule of the coordinate system; the unit vectors of each axis of $O_{M} x_{o} y_{o} z_{o}$ are given as $\mathbf{r}_{o}, \boldsymbol{\theta}_{o}$, and $\mathbf{n}_{o}$.

(4) Body coordinate $O_{M} x_{b} y_{b} z_{b}$ : the origin is the COM of the chief satellite, axis $O_{M} x_{b}$ lies along the direction of tether-span from payload $P_{2}$ to payload $P_{1}$, axis $O_{M} y_{b}$ is perpendicular to the axis $O_{M} x_{b}$ and lies in the orbital plane, and axis $O_{M} z_{b}$ follows the righthanded rule of the coordinate system. Figure 2 shows the relationship of orbit coordinate and body frame. The unit vectors of each axis of $O_{M} x_{b} y_{b} z_{b}$ are given as $\mathbf{i}_{b}, \mathbf{j}_{b}$, and $\mathbf{k}_{b}$.

In Figure $4, \theta$ is the true anomaly of the MMET, $\psi$ is the pitch angle between the projection of tether-span in orbital plane and the radius of MMET's COM, and $\phi$ is the yaw angle of the propulsion tethers.

\section{Error Dynamic Model of MMET}

In orbital plane coordinate, the position of the chief satellite $O_{M}$ and payloads $P_{1}$ and $P_{2}$ and the position of each

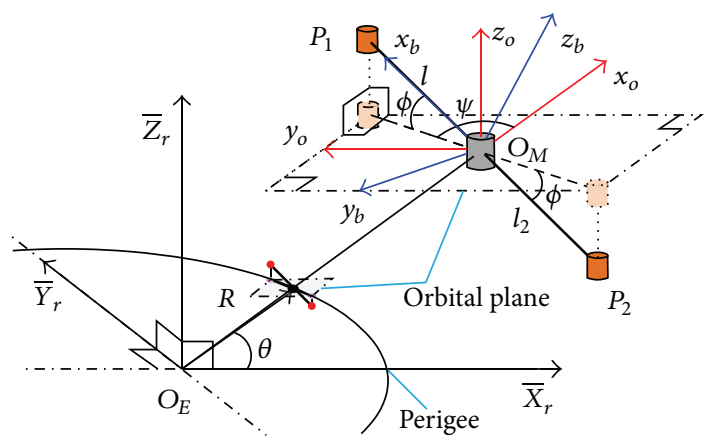

FIGURE 4: Geometry of MMET's 3D rigid model.

infinitesimal mass $d m$ of the tethers $T_{1}$ and $T_{2}$, which is $x$ apart from the MMET's COM, are as follows [17]:

$$
\begin{aligned}
\mathbf{R}= & R \cos \theta \mathbf{i}+R \sin \theta \mathbf{j}, \\
\mathbf{R}_{x_{-} T_{1}}= & (R \cos \theta+x \cos \phi \cos u) \mathbf{i} \\
& +(R \sin \theta+x \cos \phi \sin u) \mathbf{j}+x \sin \phi \mathbf{k}, \\
\mathbf{R}_{x_{-} T_{2}}= & (R \cos \theta-x \cos \phi \cos u) \mathbf{i} \\
& +(R \sin \theta-x \cos \phi \sin u) \mathbf{j}-x \sin \phi \mathbf{k}, \\
\mathbf{R}_{P_{1}}= & (R \cos \theta+l \cos \phi \cos u) \mathbf{i} \\
& +(R \sin \theta+l \cos \phi \sin u) \mathbf{j}+l \sin \phi \mathbf{k}, \\
\mathbf{R}_{P_{2}}= & {[R \cos \theta-(l+\Delta l) \cos \phi \cos u] \mathbf{i} } \\
& +[R \sin \theta-(l+\Delta l) \cos \phi \sin u] \mathbf{j} \\
& -(l+\Delta l) \sin \phi \mathbf{k},
\end{aligned}
$$

where $u=\psi+\theta, l$, and $l+\Delta l=l_{2}$ refer to the length of tethers $T_{1}$ and $T_{2}$, respectively, because the orbital plane coordinate can be treated as inertial coordinate without considering the Earth oblateness and the third-body perturbations, and so forth. So the derivatives of unit vectors of $\mathbf{i}, \mathbf{j}$, and $\mathbf{k}$ are zero; therefore, we can obtain the transverse kinetic energy $T_{\text {trans }}$ as

$$
\begin{aligned}
& T_{\text {trans }}=\frac{1}{2} M\left(\dot{R}^{2}+R^{2} \dot{\theta}^{2}\right)+\frac{1}{6} A_{2}\left(\dot{u}^{2} \cos ^{2} \phi+\dot{\phi}^{2}\right)+\frac{1}{2} \\
& \cdot A_{1}(\dot{R} \dot{u} \cos \phi \sin \psi+\dot{R} \dot{\phi} \sin \phi \cos \psi \\
& +R \dot{\theta} \dot{\phi} \sin \phi \sin \psi-R \dot{u} \dot{\theta} \cos \phi \cos \psi),
\end{aligned}
$$

where $M$ refers to the total mass of the MMET; if $m$ refers to the mass of the payload $P_{1}$ and $m+\Delta m$ refers to the mass of the payload $P_{2}$, then the total mass $M$ and coefficient $A_{1}$ and $A_{2}$ can be given as follows:

$$
\begin{aligned}
& M=m_{M}+2 m+\Delta m+\rho A(2 l+\Delta l), \\
& A_{1}=2(m \Delta l+l \Delta m+\Delta m \Delta l)+\rho A\left(2 l \Delta l+\Delta l^{2}\right),
\end{aligned}
$$




$$
\begin{aligned}
A_{2}= & 3\left[m l^{2}+(m+\Delta l)(l+\Delta l)^{2}\right] \\
& +\rho A\left[l^{3}+(l+\Delta l)^{3}\right],
\end{aligned}
$$

where $\rho$ refers to the tether's density, $A$ refers to the crosssection area of the tethers, and $m_{M}$ refers to the mass of the chief satellite. It is presumed that the configurations of payloads $P_{1}$ and $P_{2}$, the chief satellite $O_{M}$, and the tethers $T_{1}$ and $T_{2}$ are cylinders with radii $\left(r_{P}, r_{M}, r_{T}\right)$, the heights of payloads and the chief satellite are $\left(h_{P}, h_{M}\right)$, and the rotational kinetic energy of the MMET is given as

$$
T_{\text {rot }}=\frac{1}{2}\left(I_{X} \dot{\gamma}^{2}+I_{Y} \dot{\phi}^{2}+I_{Z} \dot{u}^{2}\right),
$$

where $I_{X}, I_{Y}$, and $I_{Z}$ refer to the moments of inertia of the MMET in each axis and are expressed as follows:

$$
\begin{aligned}
I_{X}= & \frac{1}{12}(2 m+\Delta m)\left(3 r_{P}^{2}+h_{P}^{2}\right)+\frac{1}{12} m_{M}\left(3 r_{M}^{2}+h_{M}^{2}\right) \\
& +\frac{\rho A}{2}(2 l+\Delta l) r_{T}^{2}, \\
I_{Y}= & \frac{1}{12}(2 m+\Delta m)\left(3 r_{P}^{2}+h_{P}^{2}\right)+\frac{1}{12} m_{M}\left(3 r_{M}^{2}+h_{M}^{2}\right) \\
& +\frac{\rho A}{12}\left\{l\left(3 r_{T}^{2}+l^{2}\right)+(l+\Delta l)\left[3 r_{T}^{2}+(l+\Delta l)^{2}\right]\right\}, \\
I_{Z}= & \frac{1}{2}(2 m+\Delta m) r_{P}^{2}+\frac{1}{2} m_{M} r_{M}^{2}+\frac{\rho A l}{12}\left(3 r_{T}^{2}+l^{2}\right) \\
& +\frac{\rho A(l+\Delta l)}{12}\left[3 r_{T}^{2}+(l+\Delta l)^{2}\right] .
\end{aligned}
$$

Combining (2) with (4), the total kinetic energy of the MMET denoted as $T$ is given by

$$
\begin{aligned}
T & =\frac{1}{6}\left\{3 M\left(\dot{R}^{2}+R^{2} \dot{\theta}^{2}\right)+A_{2}\left(\dot{u}^{2} \cos ^{2} \phi+\dot{\phi}^{2}\right)\right. \\
& +3 A_{1}[(\dot{R} \sin \psi-R \dot{\theta} \cos \psi) \dot{u} \cos \phi \\
& +(\dot{R} \cos \psi+R \dot{\theta} \sin \psi) \dot{\phi} \sin \phi]+3\left(I_{X} \dot{\gamma}^{2}+I_{Y} \dot{\phi}^{2}\right. \\
& \left.\left.+I_{Z} \dot{u}^{2}\right)\right\}
\end{aligned}
$$

where $\gamma$ refers to the rolling angle, which is the angle between the torque-plane and the "tether-spin-plane," and is defined in [18]. The next step is to deduce the potential energy $V$ of the MMET, without considering the elastic energy, so the potential energy is made up only by gravitation; from Figure 2, the potential energy of components of the MMET is given as follows:

$$
\begin{aligned}
& V_{M}=-\mu \frac{m_{M}}{R}, \\
& V_{P_{1}}=-\mu \frac{m}{\sqrt{R^{2}+l^{2}+2 R l \cos \psi \cos \phi}},
\end{aligned}
$$

$$
\begin{aligned}
& V_{P_{2}}=-\mu \frac{m+\Delta m}{\sqrt{R^{2}+(l+\Delta l)^{2}-2 R(l+\Delta l) \cos \psi \cos \phi}}, \\
& V_{T_{1}}=-\mu \rho A \int_{0}^{l} \frac{d x}{\sqrt{R^{2}+x^{2}+2 R x \cos \psi \cos \phi}}, \\
& V_{T_{2}}=-\mu \rho A \int_{0}^{l+\Delta l} \frac{d x}{\sqrt{R^{2}+x^{2}-2 R x \cos \psi \cos \phi}},
\end{aligned}
$$

where $\mu$ refers to the Earth gravitation constant. To simplify the Lagrange equations without affecting the qualitative accuracy of the results, with the method of Taylor's series expansion, and overlook higher order items of $(x / R)^{2}$ and $(l / R)^{2}$, the potential energy $V$ is given by

$$
\begin{gathered}
V=-\mu\left[\frac{M}{R}-\frac{A_{1} \cos \psi \cos \phi}{2 R^{2}}\right. \\
\left.-\frac{A_{2}\left(3 \cos ^{2} \psi \cos ^{2} \phi-1\right)}{6 R^{3}}\right] .
\end{gathered}
$$
by

Combining (6) with (8), the Lagrange function $L$ is given

$$
L=T-V \text {. }
$$

Choosing $(R, \theta, \psi, \phi, \gamma)$ as the generalized coordinates with generalized forces $\left(0,0, Q_{\psi}, Q_{\phi}, 0\right)$, the expressions of $Q_{\psi}, Q_{\phi}$ are expressed as follows [19]:

$$
\begin{aligned}
Q_{\psi} & =\tau \cos \gamma \cos \phi \\
Q_{\phi} & =\tau \sin \gamma
\end{aligned}
$$

where $\tau$ refers to the external torque acting on the propulsion tether and is generated by the rotor.

Then using Lagrange equations,

$$
\frac{d}{d t}\left(\frac{\partial L}{\partial \dot{q}_{i}}\right)-\frac{\partial L}{\partial q_{i}}=Q_{i}
$$

We can obtain the $3 \mathrm{D}$ error dynamics of the MMET as follows:

$$
\begin{aligned}
\ddot{R} & -R \dot{\theta}^{2}+\frac{\mu}{R^{2}}+\frac{\mu A_{1} \cos \psi \cos \phi}{M R^{3}} \\
& +\frac{\mu A_{2}\left(3 \cos ^{2} \psi \cos ^{2} \phi-1\right)}{2 M R^{4}} \\
& +\frac{A_{1}}{2 M}\left[\left(\dot{u}^{2}+\dot{\phi}^{2}\right) \cos \psi \cos \phi-2 \dot{u} \dot{\phi} \sin \psi \sin \phi\right. \\
& +\ddot{u} \sin \psi \cos \phi+\ddot{\phi} \cos \psi \sin \phi]=0,
\end{aligned}
$$




$$
\begin{aligned}
6 & R^{2} \ddot{\theta}+6 I_{Z} \ddot{u}+2 A_{2} \ddot{u} \cos ^{2} \phi+12 M R \dot{R} \dot{\theta}-2 A_{2} \dot{u} \dot{\phi} \sin 2 \phi \\
& +3 A_{1}[-(2 \dot{R} \dot{\theta}+2 R \ddot{\theta}+R \ddot{\psi}) \cos \psi \cos \phi \\
& +\left(2 R \dot{\psi} \dot{\theta}+R \dot{\psi}^{2}+R \dot{\phi}^{2}+\ddot{R}\right) \sin \psi \cos \phi \\
& +2 R \dot{\psi} \dot{\phi} \cos \psi \sin \phi+R \ddot{\phi} \sin \psi \sin \phi]=0, \\
\ddot{u}+ & \frac{A_{2}}{3 I_{Z}}\left(\ddot{u} \cos ^{2} \varphi-\dot{u} \dot{\phi} \sin 2 \phi\right)+\frac{A_{2} \mu \cos ^{2} \phi \sin 2 \psi}{2 I_{Z} R^{3}} \\
& +\frac{A_{1} \mu \cos \phi \sin \psi}{2 I_{Z} R^{2}}+\frac{A_{1}}{2 I_{Z}}[-(2 \dot{R} \dot{\theta}+R \ddot{\theta}) \cos \psi \cos \phi \\
& \left.+\left(\ddot{R}-R \dot{\theta}^{2}\right) \sin \psi \cos \phi\right]=\frac{\tau \cos \gamma \cos \phi}{I_{Z}}, \\
\ddot{\phi}+ & \frac{3 A_{1}\left[(R \ddot{\theta}+2 \dot{R} \dot{\theta}) \sin \psi \sin \phi+\left(\ddot{R}-R \dot{\theta}^{2}\right) \cos \psi \sin \phi\right]}{2 A_{2}+6 I_{Y}} \\
& +\frac{A_{2} \dot{u}^{2} \sin 2 \phi}{\left(2 A_{2}+6 I_{Y}\right)}+\frac{3 A_{2} \mu \cos ^{2} \psi \sin 2 \phi}{\left(2 A_{2}+6 I_{Y}\right) R^{3}} \\
& +\frac{3 A_{1} \mu \cos \psi \sin \phi}{\left(2 A_{2}+6 I_{Y}\right) R^{2}}=\frac{\tau \sin \gamma}{A_{2} / 3+I_{Y}}, \\
I_{X} \ddot{\gamma} & =0 .
\end{aligned}
$$

\section{Attitude and Orbit Coupling Analysis Considering Structural Bias}

As we know, when the asymmetries caused by tether length or payload mass exist, the COM of the MMET will deviate from the COM of the chief satellite to other places. If we denote the MMET's COM as point $C$, the radius of the two points relative to the Earth's COM is $\mathbf{R}$ and $\mathbf{R}_{C}$; the schematic is shown in Figure 5.

As we know, if the two payloads have the same masses and the two tethers have the same lengths simultaneously, then point $O_{M}$ and point $C$ will coincide with each other; in other words, $\mathbf{r}_{C}=\mathbf{0}$. However, while the difference in tethers' length and the difference in payloads' mass exist, the COM's deviation $\mathbf{r}_{C} \neq \mathbf{0}$. The MMET's COM orbital dynamic equation in two-body system $[19,20]$ is described by

$$
\ddot{\mathbf{R}}_{C}=-\frac{\mu}{R_{C}^{3}} \mathbf{R}_{C}
$$

where the vector $\mathbf{R}_{C}$ can be expressed by $\mathbf{R}$ and COM deviation vector $\mathbf{r}_{C}$ as follows:

$$
\mathbf{R}+\mathbf{r}_{C}=\mathbf{R}_{C}
$$

Combining (13) and (14), the dynamic equations of the chief satellite are obtained by $\left(r_{C} \ll R\right)$

$$
\ddot{\mathbf{R}}=-\frac{\mu}{R^{3}} \mathbf{R}-\frac{\mu}{R^{3}}\left[\mathbf{r}_{C}-\frac{3 r_{C}}{R}\left(\mathbf{R}+\mathbf{r}_{C}\right)\right]-\ddot{\mathbf{r}}_{C},
$$

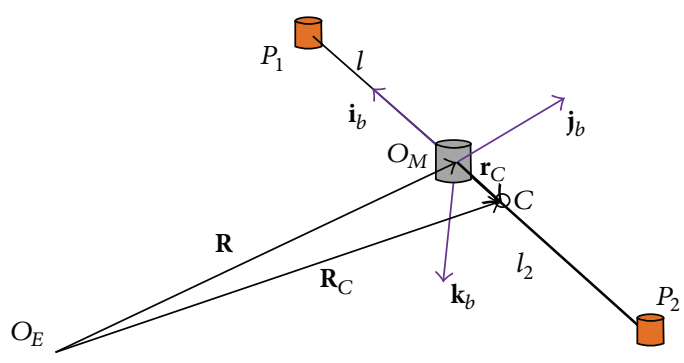

FIGURE 5: The schematic of MMET with structure bias.

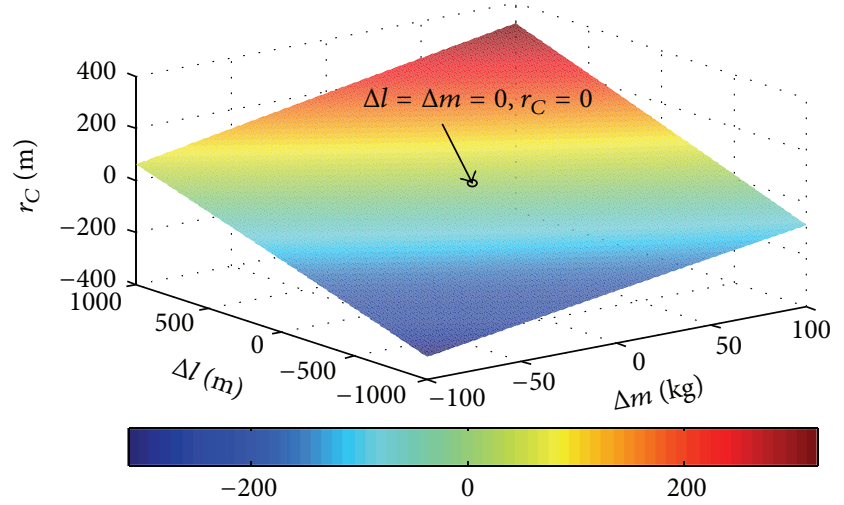

Figure 6: The COM deviation of MMET mesh.

where the COM deviation $\mathbf{r}_{C}$ of the MMET is the functions of $\Delta l$ and $\Delta m$ and is expressed by [21]

$$
\begin{aligned}
\mathbf{r}_{C} & =-\left(\frac{m \Delta l+l \Delta m+\Delta m \Delta l+\rho A l \Delta l+(1 / 2) \rho A \Delta l^{2}}{m_{M}+2 m+\Delta m+\rho A(2 l+\Delta l)}\right) \\
& \cdot \mathbf{i}_{b} .
\end{aligned}
$$

Figure 6 shows the relationship of $r_{C}$ with the difference in tethers' length and the difference in payloads' mass. The other parameters are listed in Table 2. It is shown that, with the increase of $\Delta l$ and $\Delta m$, the COM deviation $r_{C}$ increases linearly.

If we denote $\mathbf{F}_{C}$ as

$$
\frac{\mathbf{F}_{C}}{M}=-\frac{\mu}{R^{3}}\left[\mathbf{r}_{C}-\frac{3 r_{C}}{R}\left(\mathbf{R}+\mathbf{r}_{C}\right)\right]-\ddot{\mathbf{r}}_{C}
$$

which is the external centrifugal force acting on the chief satellite caused by the tether length deviation $\Delta l$ and payload mass deviation $\Delta m$. What is more, without considering the rolling angle, the rotational angular velocity $\omega_{b}$ in the body coordinate relative to the orbit coordinate $O_{M} x_{o} y_{o} z_{o}$ is given by

$$
\begin{aligned}
\boldsymbol{\omega}_{b} & =\left[\begin{array}{c}
0 \\
-\dot{\phi} \\
0
\end{array}\right]+\left[\begin{array}{ccc}
\cos \phi & 0 & \sin \phi \\
0 & 1 & 0 \\
-\sin \phi & 0 & \cos \phi
\end{array}\right]\left[\begin{array}{l}
0 \\
0 \\
\dot{\psi}
\end{array}\right] \\
& =\dot{\psi} \sin \phi \cdot \mathbf{r}_{o}-\dot{\phi} \cdot \boldsymbol{\theta}_{o}+\dot{\psi} \cos \phi \cdot \mathbf{n}_{o} .
\end{aligned}
$$


TABLE 1: The change range of $\mathbf{F}_{C}$ with difference in tethers' length and different external torque value.

\begin{tabular}{lcccc}
\hline $\mathbf{F}_{C}(\mathrm{~N})$ & $\Delta l=0.01 \mathrm{~m}$ & $\Delta l=0.1 \mathrm{~m}$ & $\Delta l=1 \mathrm{~m}$ & $\Delta l=10 \mathrm{~m}$ \\
\hline$\tau=0 \mathrm{~N} \cdot \mathrm{m}$ & {$[0.1251,0.1206]$} & {$[1.251,1.206]$} & {$[12.51,12.11]$} & {$[126.3,120.5]$} \\
$\tau=25 \mathrm{kN} \cdot \mathrm{m}$ & {$[0.133,0.1217]$} & {$[1.33,1.217]$} & {$[13.32,12.18]$} & {$[134.2,122]$} \\
$\tau=250 \mathrm{kN} \cdot \mathrm{m}$ & {$[0.2215,0.1228]$} & {$[2.214,1.228]$} & {$[22.21,12.28]$} & {$[220.8,122.8]$} \\
\hline
\end{tabular}

Then, the components of $\ddot{\mathbf{r}}_{C}$ in the orbit coordinate $O_{M} x_{o} y_{o} z_{o}$ are obtained by

$$
\ddot{\mathbf{r}}_{C}=\mathbf{r}_{C}^{\prime \prime}+2 \boldsymbol{\omega}_{b} \times \mathbf{r}_{C}^{\prime}+\boldsymbol{\omega}_{b}^{\prime} \times \mathbf{r}_{C}+\boldsymbol{\omega}_{b} \times\left(\boldsymbol{\omega}_{b} \times \mathbf{r}_{C}\right),
$$

where the symbols " " " " and "' " refer to the relative two-order and one-order derivative in the body frame $O_{M} x_{b} y_{b} z_{b}$. The expanded form of (19) is given by

$$
\ddot{\mathbf{r}}_{C}=\left[\begin{array}{c}
r_{C}\left(\omega_{b y}^{2}+\omega_{b z}^{2}\right)-\ddot{r}_{C} \\
-r_{C} \dot{\omega}_{b z}-2 \dot{r}_{C} \omega_{b z}-r_{C} \omega_{b x} \omega_{b y} \\
r_{C} \dot{\omega}_{b y}+2 \dot{r}_{C} \omega_{b y}-r_{C} \omega_{b x} \omega_{b z}
\end{array}\right]
$$

Furthermore, the components of $\mathbf{R}$ in the orbit coordinate are $[R, 0,0]$ and the components of $\mathbf{r}_{C}$ in the movement frame are $\left[-r_{C} \cos \psi,-r_{C} \sin \psi, 0\right]$. As we know, the differences in tether's length $\Delta l$ and in payloads' mass deviation are constant to the MMET. So the derivatives of $r_{C}$ (one-order and twoorder) are zero in body frame. So (20) is simplified to

$$
\ddot{\mathbf{r}}_{C}=r_{C}\left[\begin{array}{c}
\omega_{b y}^{2}+\omega_{b z}^{2} \\
-\dot{\omega}_{b z}-\omega_{b x} \omega_{b y} \\
\dot{\omega}_{b y}-\omega_{b x} \omega_{b z}
\end{array}\right] .
$$

Substituting (21) into (17) yields

$$
\begin{aligned}
& \frac{\mathbf{F}_{C}}{M} \\
& =r_{C}\left\{\begin{array}{c}
\frac{\mu}{R^{3}}\left[3+\left(1-\frac{3 r_{C}}{R}\right) \cos \psi\right]-\left(\omega_{b y}^{2}+\omega_{b z}^{2}\right) \\
\frac{\mu}{R^{3}}\left(1-\frac{3 r_{C}}{R}\right) \sin \psi+\dot{\omega}_{b z}+\omega_{b x} \omega_{b y} \\
\omega_{b x} \omega_{b z}-\dot{\omega}_{b y}
\end{array}\right\} .
\end{aligned}
$$

It is shown from (22) that while the yaw angle $\phi=$ $0 \mathrm{rad}$, the movements of the propulsion tether are restricted in the orbital plane, and the component of the centrifugal acceleration perpendicular to the orbital plane becomes zero; we can see that the rotational movement of tether without considering the yaw angle has no influence on the orientation of the orbital plane, which is only affected by the pitch angle $\psi$ and its angular velocity $\dot{\psi}$. However, if the yaw angle $\phi \neq 0$, the component of $\mathbf{F}_{C}$ perpendicular to the orbital plane exists and the out-of-plane movement will affect the orbit of the MMET (especially the orientation of the orbit).

Figures 7 and 8 show the relation between the external centrifugal force $\mathbf{F}_{C}$ and revs of the propulsion tether, under the conditions of different external torque $\tau$. We can observe that while the external torque is zero, the external centrifugal
TABLE 2: Parameters of the MMET.

\begin{tabular}{lcc}
\hline Parameters & Value & Unit \\
\hline Earth's gravitation constant: $\mu$ & $3.9877848 \times 10^{14}$ & $\mathrm{~m}^{3} / \mathrm{s}^{2}$ \\
Length of propulsion tether $T_{1}: l$ & 10000 & $\mathrm{~m}$ \\
Mass of chief satellite: $m_{M}$ & 5000 & $\mathrm{~kg}$ \\
Mass of payload $P_{1}: m$ & 1000 & $\mathrm{~kg}$ \\
The density of tethers: $\rho$ & 970 & $\mathrm{~kg} / \mathrm{m}^{3}$ \\
Cross-section area of tethers: $A$ & $6.283 \times 10^{-5}$ & $\mathrm{~m}^{2}$ \\
The radius of chief satellite: $r_{M}$ & 0.5 & $\mathrm{~m}$ \\
The height of chief satellite: $h_{M}$ & 1.5 & $\mathrm{~m}$ \\
The radius of payloads: $r_{P}$ & 0.5 & $\mathrm{~m}$ \\
The height of payloads: $h_{P}$ & 1.5 & $\mathrm{~m}$ \\
The radius of the tether: $r_{T}$ & 0.00447207 & $\mathrm{~m}$ \\
\hline
\end{tabular}

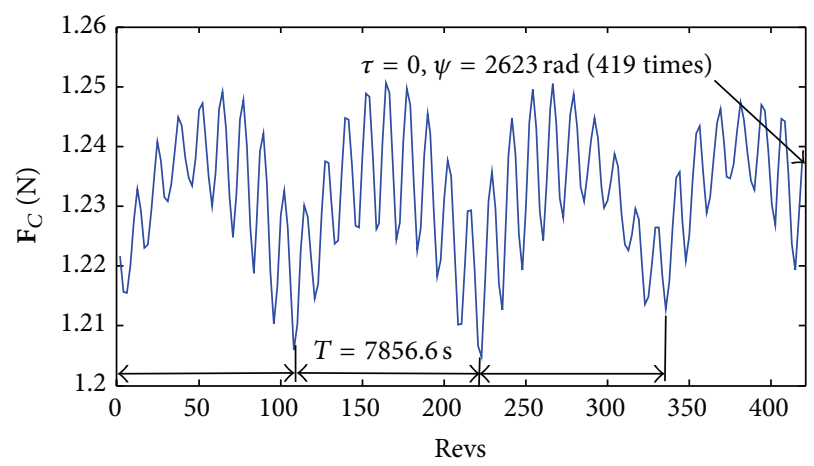

Figure 7: The relationship between $\mathbf{F}_{C}$ and revs of propulsion tether with $\Delta l=0.1 \mathrm{~m}$ and $\tau=0$.

force $\mathbf{F}_{C}$ oscillates around the initial value (the minimum value is $1.205 \mathrm{~N}$ and the maximum value is $1.251 \mathrm{~N}$ ). More specifically, the change period of $\mathbf{F}_{C}$ coincides with the orbit period of the MMET. When the MMET reaches the perigee, $\mathbf{F}_{C}$ gets its maximum value; reversely, when the MMET reaches the apogee, $\mathbf{F}_{C}$ gets its minimum value. From Figure 8 , we can see that while the external torque is nonzero, the magnitude of $\mathbf{F}_{C}$ varies with the revs of the propulsion tether increasingly. What is more, from Figure 8 it is also shown that the greater the external torque value is, the faster the magnitude of $\mathbf{F}_{C}$ increases. Additionally, when the tether length deviation $\Delta l$ increases, the change trend of $\mathbf{F}_{C}$ is similar to the change trend of $\mathbf{F}_{C}$ shown in Figures 7 and 8 but has different magnitude.

Table 1 shows the change range of $\mathbf{F}_{C}$ with different $\Delta l$ and external torque $\tau$. It is shown that while the external torque has the same value, with the tether length deviation increasing from $0.01 \mathrm{~m}$ to $10 \mathrm{~m}$, the range of $\mathbf{F}_{C}$ increases correspondingly. Reversely, while the tether length has the 


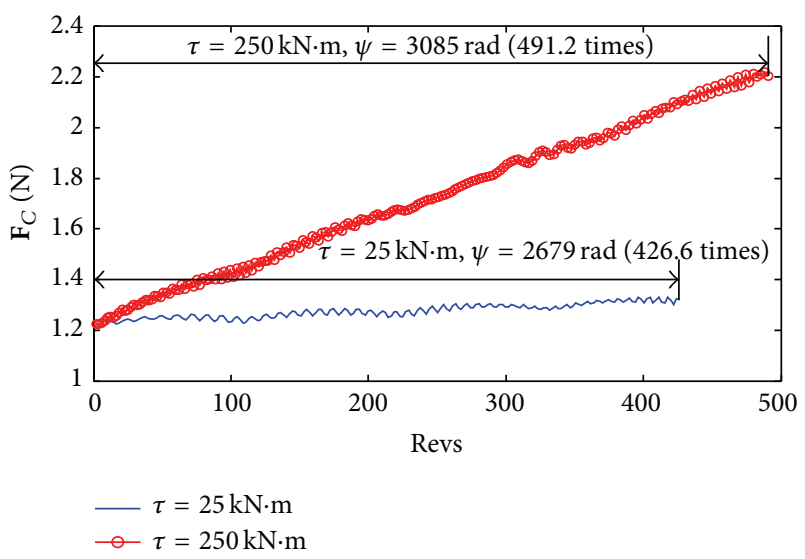

FIGURE 8: The relationship between $\mathbf{F}_{C}$ and revs of the propulsion tether with $\Delta l=0.1 \mathrm{~m}$ and different $\tau$.

same deviation, with increasing the external torque, the range of $\mathbf{F}_{C}$ increases nonlinearly and slowly.

\section{Numerical Simulations}

In order to quantify the relationship of structural bias and the dynamic characteristics of the MMET, this section presents the numerical simulations of the dynamics model by MATLAB. Table 2 shows the initial parameters of the MMET.

Furthermore, the initial values of the generalized coordinates and generalized velocities are given as follows:

$$
\begin{aligned}
& R(0)=6.728 \times 10^{6} \mathrm{~m}, \\
& \dot{R}(0)=0 \mathrm{~m} / \mathrm{s}, \\
& \theta(0)=0 \mathrm{rad}, \\
& \dot{\theta}(0)=0.00126 \mathrm{rad} / \mathrm{s}, \\
& \psi(0)=0 \mathrm{rad}, \\
& \dot{\psi}(0)=0.0873 \mathrm{rad} / \mathrm{s}, \\
& \phi(0)=0.1 \mathrm{rad}, \\
& \dot{\phi}(0)=0 \mathrm{rad} / \mathrm{s}, \\
& \gamma(0)=0 \mathrm{rad}, \\
& \dot{\gamma}(0)=0 \mathrm{rad} / \mathrm{s} .
\end{aligned}
$$

Figure 9 shows the time history of the radius of the chief satellite's COM with different $\Delta l$ (from 0 to $10 \mathrm{~m}$ ). It is shown that, from the macroscopic view, the COM of the chief satellite varies periodically over time, and the orbit period $T=$ 7856.6 s. Note that there is no evident effect of the difference in tethers' length on the radius of the chief satellite when $\Delta l$ is very small $\left(r_{C} \ll R\right)$. However, Figure 10 shows that the magnitude of the difference $\Delta R$ between the MMET's radius of $3 \mathrm{D}$ error model and $3 \mathrm{D}$ ideal model increases exponentially with $\Delta l$ increasing; $\Delta R$ is defined by

$$
\Delta R=R_{a}-R_{b},
$$

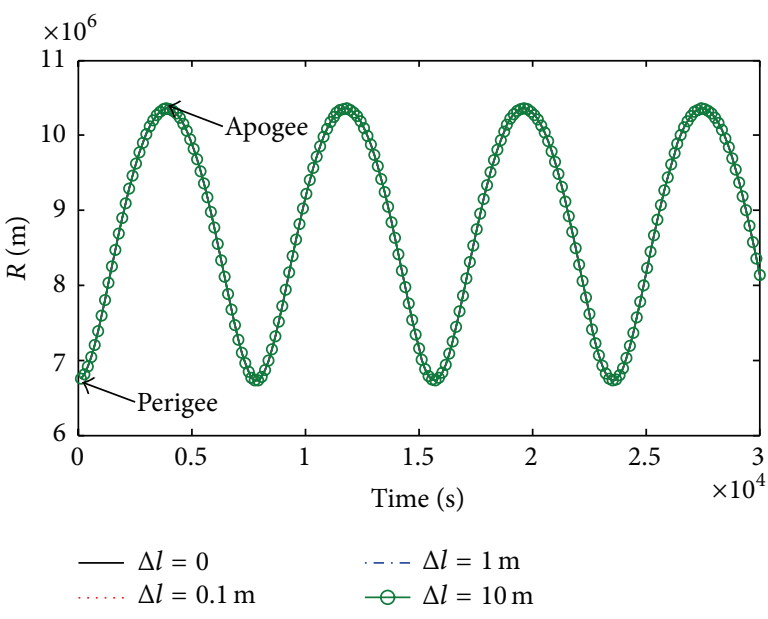

Figure 9: Time history of $R$ with different $\Delta l, \tau=250 \mathrm{kN} \cdot \mathrm{m}$.

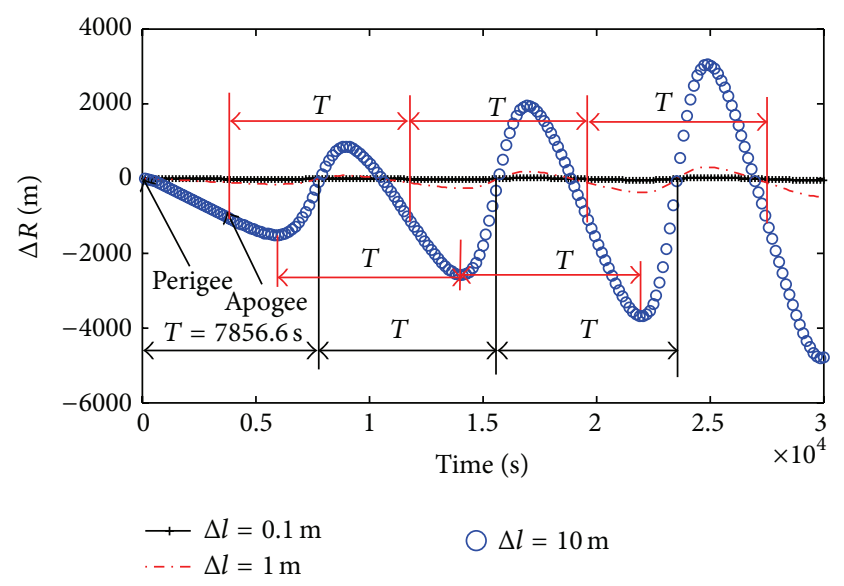

FIgURE 10: Time history of $\Delta R$ with different $\Delta l, \tau=250 \mathrm{kN} \cdot \mathrm{m}$.

where the subscript " $a$ " refers to "asymmetry" and $R_{a}$ stands for the radius of the chief satellite with asymmetries in tether length and payload mass, the subscript " $b$ " refers to "balance," and $R_{b}$ refers to the radius of the chief satellite with symmetrical structure. From Figure 10, we can get that while increasing $\Delta l$ from $0.1 \mathrm{~m}$ to $10 \mathrm{~m}$, the peak of each period of $\Delta R$ increases exponentially. From this view, if the MMET orbits the Earth for several days or months, the radius deviation $\Delta R$ caused by the asymmetries will get a huge value and be hard to control. Additionally, we can observe from Figure 10 that the perigee and apogee of the orbit of the chief satellite are unchanged over time if the tether length deviation $\Delta l$ is unchanged. However, when $\Delta l \neq 0$ or $\Delta m \neq 0$, the apogee of the chief satellite has changed slightly compared to the case that $\Delta l=0$ and $\Delta m=0$. Table 3 shows the relation between the orbit elements (including semimajor axis $a$, eccentricity $e$, and semilatus rectum $p$ ) and $\Delta l$. It is shown that the existence of the asymmetries will change the orbit elements weakly. However, the orbit elements will be unchanged over time.

Figure 11 shows the time history of the true anomaly $\theta$ with different tether length deviation $\Delta l$. It is illustrated that 
TABLE 3: The relative orbital errors with difference in tethers' length.

\begin{tabular}{lcccc}
\hline Structure bias & $r_{a}\left(\times 10^{6} \mathrm{~m}\right)$ & $a\left(\times 10^{6} \mathrm{~m}\right)$ & $e$ & $p\left(\times 10^{6} \mathrm{~m}\right)$ \\
\hline$\Delta l=0$ & 10.360000 & 8.5440000 & 0.2125468 & 8.1580150 \\
$\Delta l=0.1 \mathrm{~m}$ & 10.359989 & 8.5439945 & 0.2125463 & 8.1580116 \\
$\Delta l=1 \mathrm{~m}$ & 10.359892 & 8.5439460 & 0.2125418 & 8.1579816 \\
$\Delta l=10 \mathrm{~m}$ & 10.358919 & 8.5434595 & 0.2124970 & 8.1576798 \\
\hline
\end{tabular}

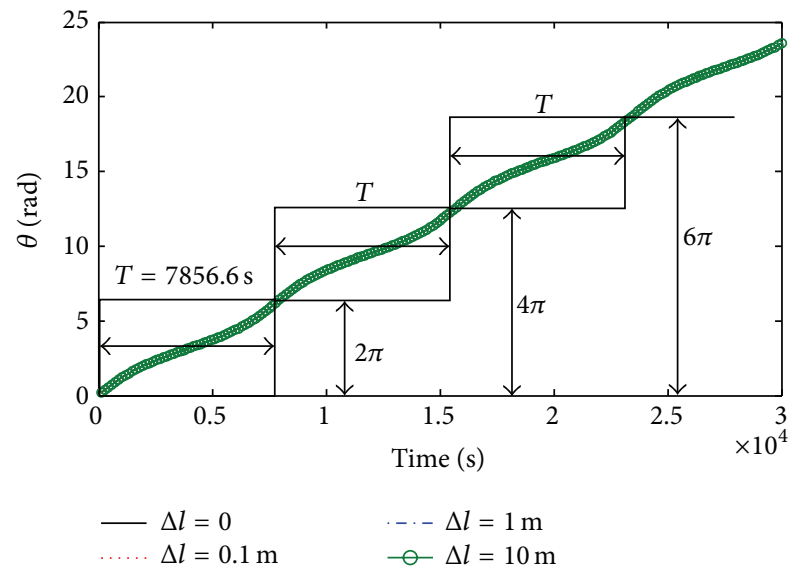

FIgURE 11: Time history of $\theta$ with different $\Delta l, \tau=250 \mathrm{kN} \cdot \mathrm{m}$.

$\theta$ increases linearly with minor wiggles over time. This is because the fact that when the MMET orbits Earth from perigee to apogee, the distance of the MMET from the Earth $\mathrm{COM}$ increases over time; the result is that the angular velocity $\dot{\theta}$ decreases correspondingly. Reversely, when the MMET moves from the apogee to perigee, the angular velocity $\dot{\theta}$ increases nonlinearly.

Furthermore, from Figure 10, we can also observe that, in each period, there is a critical point, where $\Delta R=0$. Before this point, $\Delta R>0$, it means that the COM of the chief satellite with asymmetrical structure $(\Delta l \neq 0, \Delta m \neq 0)$ is farther than the COM of the chief satellite with symmetrical structure $(\Delta l=\Delta m=0)$ simultaneously; the result is that the MMET with asymmetrical structure moves slower than the MMET with symmetrical structure, so $\Delta \theta$ decreases nonlinearly. After that point, $\Delta R<0$, the MMET with asymmetrical structure moves faster than the MMET with symmetrical structure; then, $\Delta \theta$ increases nonlinearly until returning back to the perigee; the change trend of $\Delta \theta$ is shown in Figure 12.

Next, we study the influence of the difference in tethers' length $\Delta l$ on the attitude elements of the MMET, including the pitch angle $\psi$ and the yaw angle $\phi$. Under the condition of external torque $\tau=0$ and $\tau=250 \mathrm{kN} \cdot \mathrm{m}$, Figures 13 and 14 show the time history of pitch angle $\psi$ and pitch angle error $\Delta \psi$ with different $\Delta l$, respectively. It is shown that $\psi$ increases linearly while $\tau=0$ and increases exponentially while $\tau=$ $250 \mathrm{kN} \cdot \mathrm{m}$ over time. From Figure 14, we can observe that due to the tether length deviation $\Delta l$, with increasing $\Delta l, \Delta \psi$ increases nonlinearly. On the other hand, while considering the effect of the external torque, which changes the pitch angle

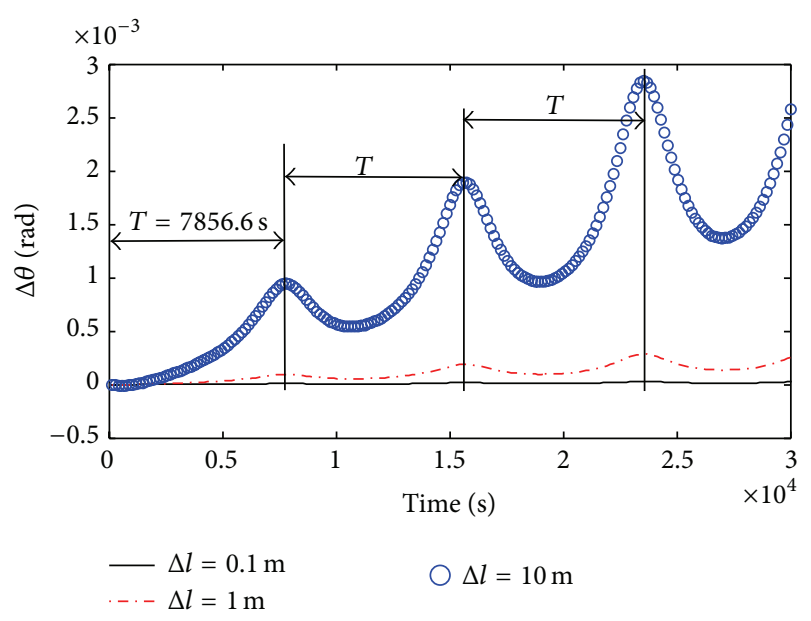

Figure 12: Time history of $\Delta \theta$ with different $\Delta l, \tau=250 \mathrm{kN} \cdot \mathrm{m}$.

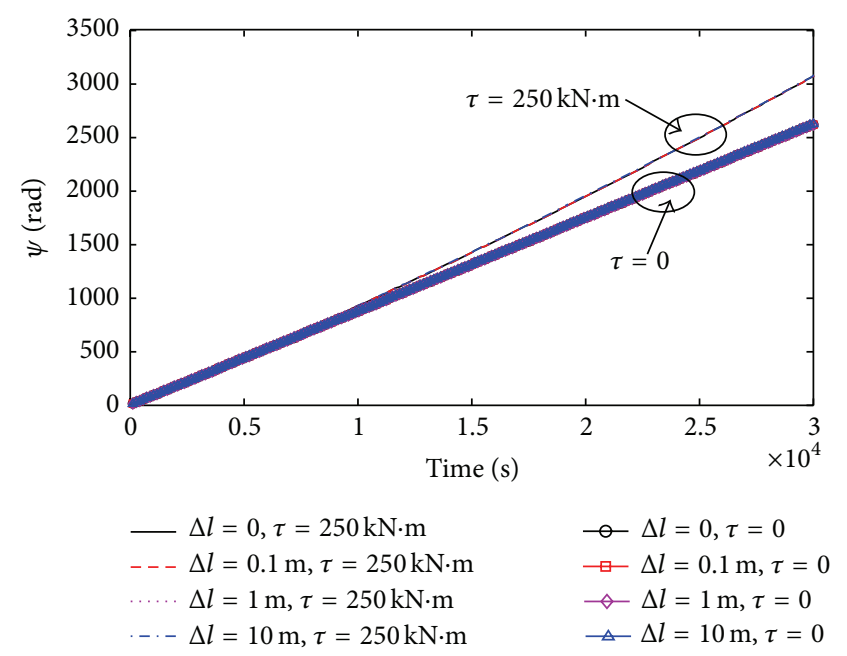

Figure 13: Time history of pitch angle $\psi$ with different $\Delta l$.

$\psi$ directly, the influence of the tether length deviation $\Delta l$ on the attitude angle $\psi$ becomes weaker than the case of $\tau=0$.

As we know, the angular velocity of the propulsion tether is the key to decide the injection precision of the payload. Therefore, whether the asymmetries of the tether length or payload mass have influence on the angular velocity $\dot{\psi}$ of the propulsion tether is very important. Figures 15 and 16 show the time history of the angular velocity $\dot{\psi}\left(=\omega_{\psi}\right)$ with different tether length deviation with different simulation steps under the same precondition of $\tau=0$. It is shown that, from the macroscopic view, increasing $\Delta l$ from $0.1 \mathrm{~m}$ to $10 \mathrm{~m}$ leads to the maximum value of $\dot{\psi}$ in each circle around the chief satellite which increases correspondingly. The result is that the rotational period of the propulsion tether decreases. Furthermore, Figure 15 shows that, in each orbit period of the MMET, the magnitude of $\dot{\psi}$ changes with high-frequency oscillation and obtains its maximum value at apogee. This is because of the existence of the gravity gradient torque, noted as $\tau_{G}$. The schematic of the gravity gradient torque with different position in the parking orbit is shown in Figure 17. 


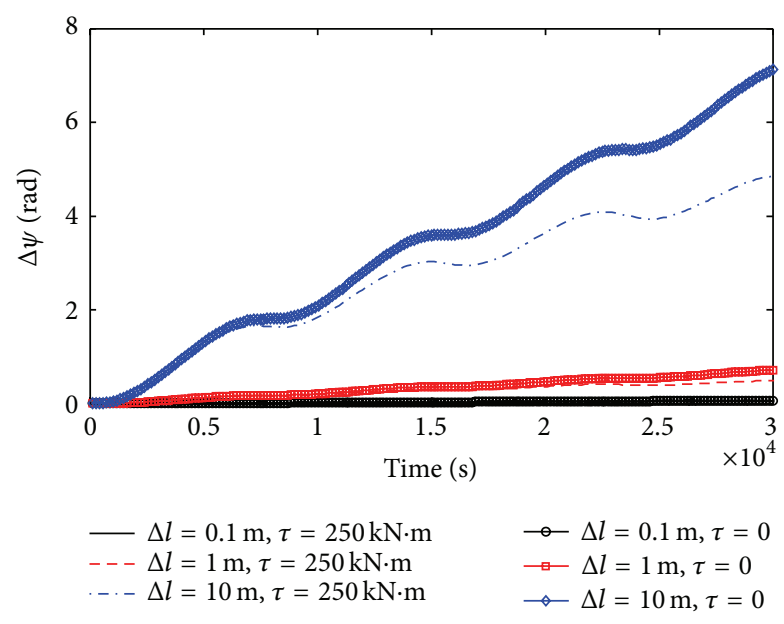

Figure 14: Time history of $\Delta \psi$ with different $\Delta l$.

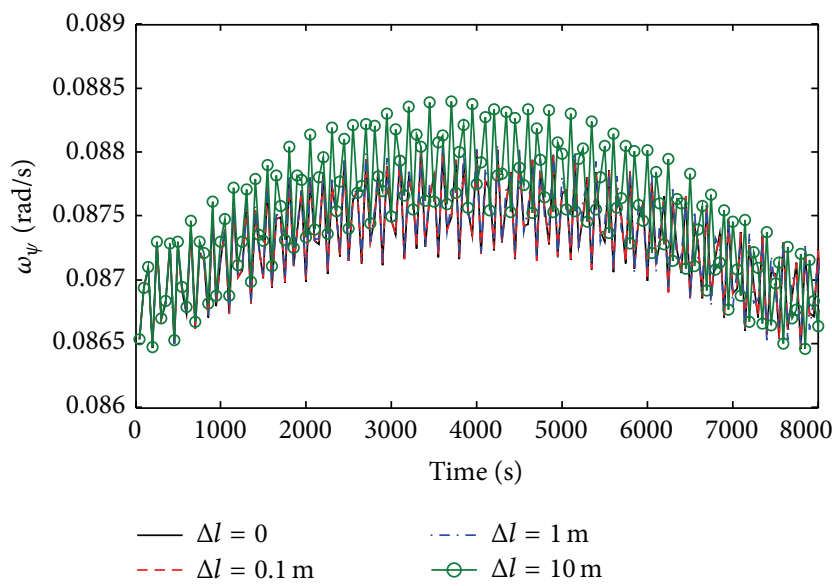

FIGURE 15: Time history of angular velocity $\dot{\psi}$ with different $\Delta l$ in an orbit period, $\tau=0$ (simulation step $=50)$.

While the gravity gradient torque has opposite direction to the rotational direction of the propulsion tether $\left(\tau_{G}<0\right), \tau_{G}$ will hinder the rotation of the propulsion tether; as a result, the pitch angular velocity $\dot{\psi}$ will decrease nonlinearly over time till $\tau_{G}=0$. After that, while $\tau_{G}>0$, the propulsion tether obtains some extra angular acceleration, which will promote the rotation of the propulsion tether, and the angular velocity $\dot{\psi}$ increases nonlinearly. The oscillation magnitude of $\dot{\psi}$ is determined by the length of the propulsion tether, meaning that the longer the tether length is, the more obvious effect of the gravity gradient torque has on the rotational angular velocity $\dot{\psi}$.

Figure 18 shows the time history of the angular acceleration $\ddot{\psi}\left(=\alpha_{\psi}\right)$ with different tether length deviation $\Delta l$. It is shown from Figure 18 that, with increasing $\Delta l$, the magnitude of pitch angular acceleration $\ddot{\psi}$ has no evident variance but has sustained oscillation over time while the external torque is zero, and the magnitude of $\ddot{\psi}$ is determined by the gravity gradient torque $\tau_{G}$. Furthermore, as mentioned above, when $\Delta l$ is small, the tether length deviation has little influence on the angular velocity $\dot{\psi}$; however, the magnitude of $\dot{\psi}$ will

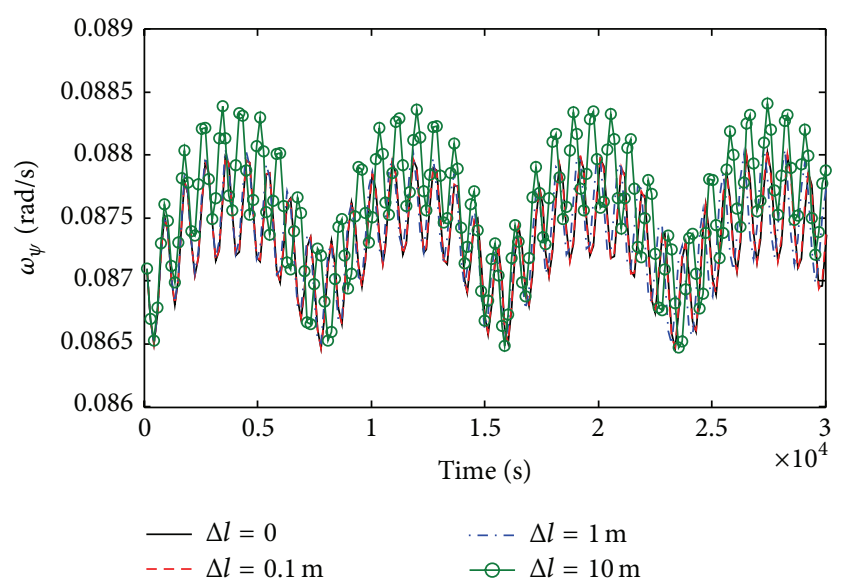

FIGURE 16: Time history of angular velocity $\dot{\psi}$ with different $\Delta l, \tau=0$ ( simulation step $=150)$.

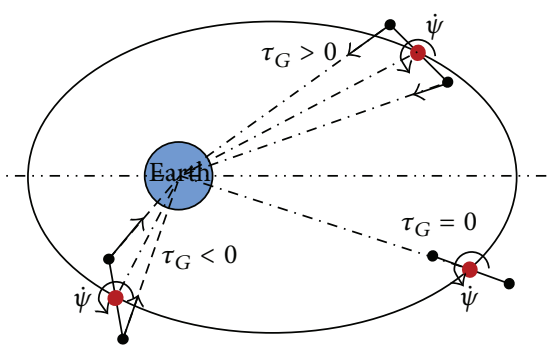

FIGURE 17: Schematic of the effect of the gravity gradient torque on the pitch angular velocity with different attitude.

increase when $\Delta l$ is greater, which causes the change of the rotation period of the propulsion tether. As a result, the magnitude of $\Delta \ddot{\psi}\left(=\Delta \alpha_{\psi}\right)$ increases linearly while $\Delta l$ is small but increases irregularly while $\Delta l$ is much greater. The result is shown in Figure 19.

As we know, the payload injection precision is determined by the position and velocity (magnitude and direction) at the perigee; therefore, out-of-plane movement of the propulsion tether is very crucial to the payload injection precision. Figure 20 shows the time history of yaw angle $\phi$ with different tether length deviation $\Delta l$. We can observe that $\phi$ changes with equal amplitude oscillations over time and the magnitude value equals the initial value of $\phi(0)$. Because of the coupling effect between the pitch angle $\psi$ and yaw angle $\phi$, the oscillation period of $\phi$ equals the rotation period of $\psi$; what is more, the oscillation period of $\phi$ decreases while $\Delta l$ increases. The yaw angular velocity $\dot{\phi}$ also changes periodically with undamped oscillation over time and is shown in Figure 22. If the external torque $\tau$ exists, the variation trend of yaw angle $\phi$ and yaw angular velocity $\dot{\phi}$ is shown in Figures 21 and 23. From these two figures, we can observe that while $\tau \neq 0$, the influences of asymmetries on the yaw angle and yaw angular velocity are weakened due to the composite effects of gravity gradient torque $\tau_{G}$ and external torque $\tau$ and coupling effects of attitude angles. The results 


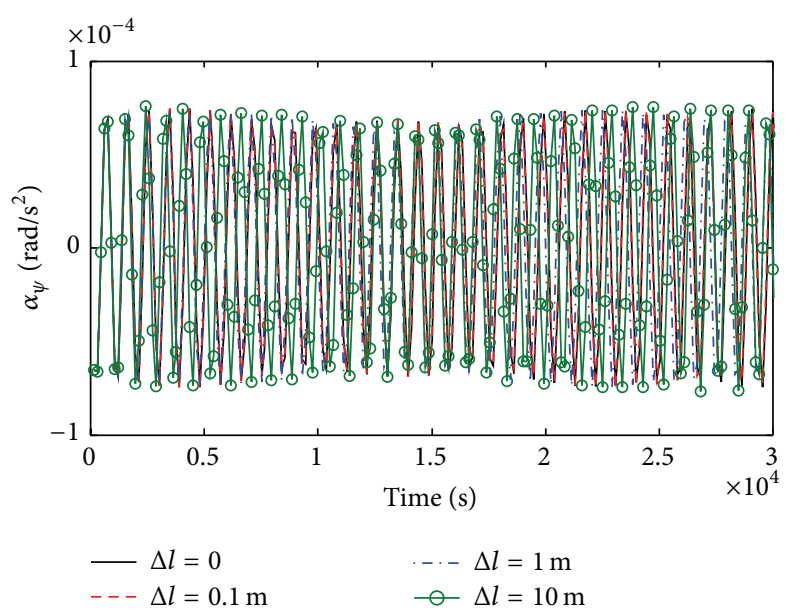

FIgURE 18: The time history of pitch angular acceleration with different $\Delta l, \tau=0$.

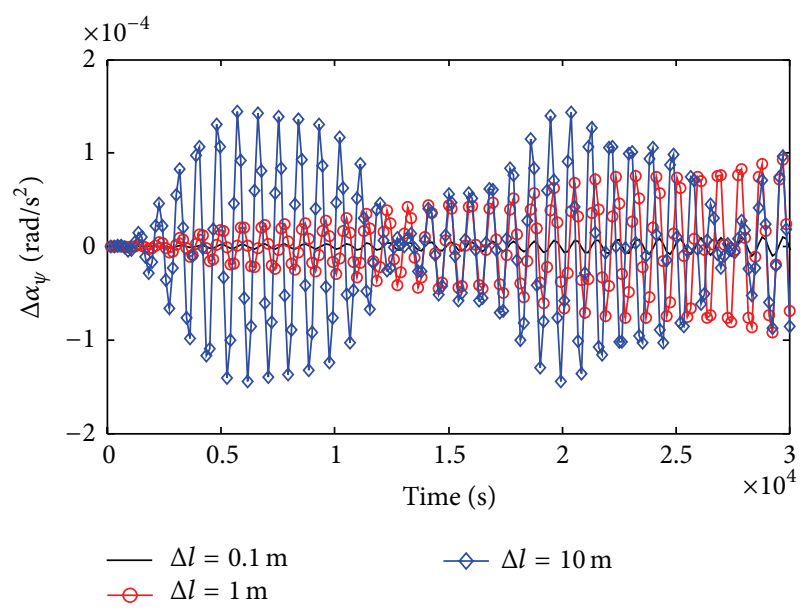

FIGURE 19: The time history of pitch angular acceleration error $\Delta \alpha_{\psi}$ with different $\Delta l, \tau=0$.

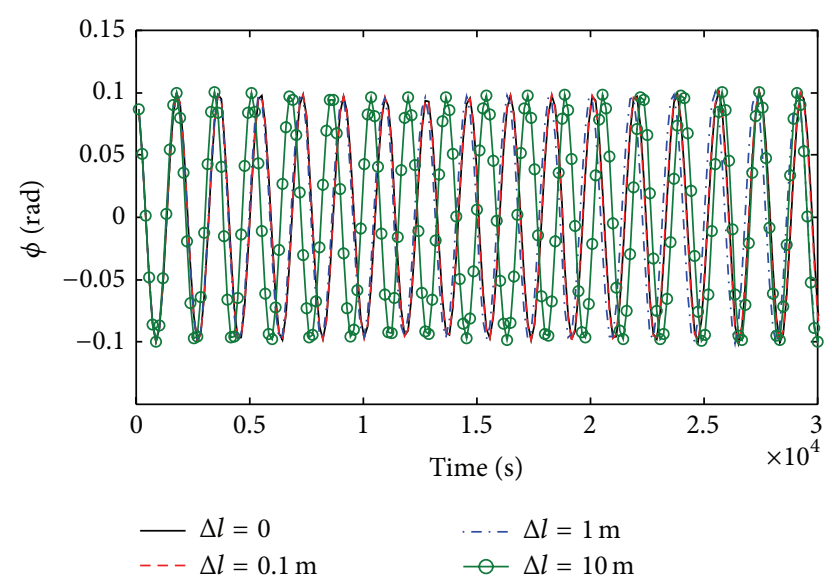

Figure 20: The time history of yaw angle $\phi$ with different $\Delta l, \tau=0$.

of those effects are that the angle $\phi$ and angular velocity $\dot{\phi}$ oscillate irregularly over time.

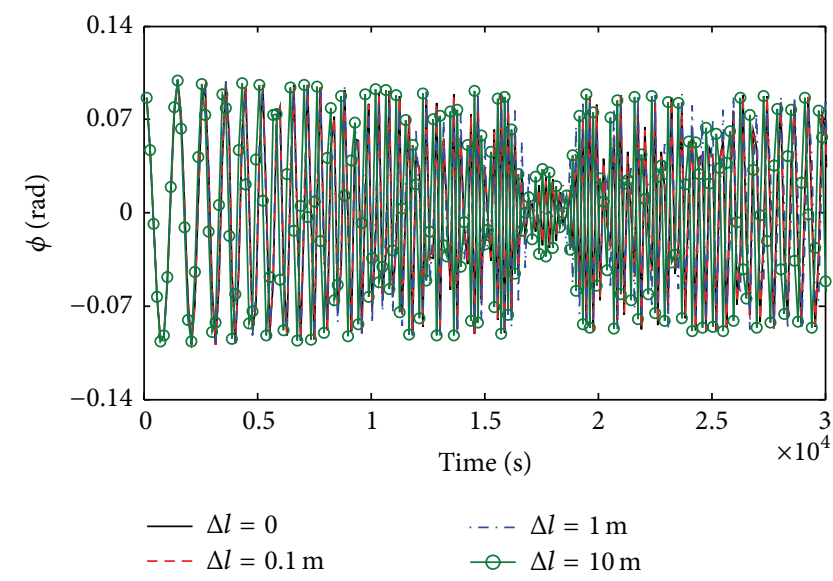

FIgURE 21: The time history of yaw angle $\phi$ with different $\Delta l, \tau=$ $250 \mathrm{kN} \cdot \mathrm{m}$.

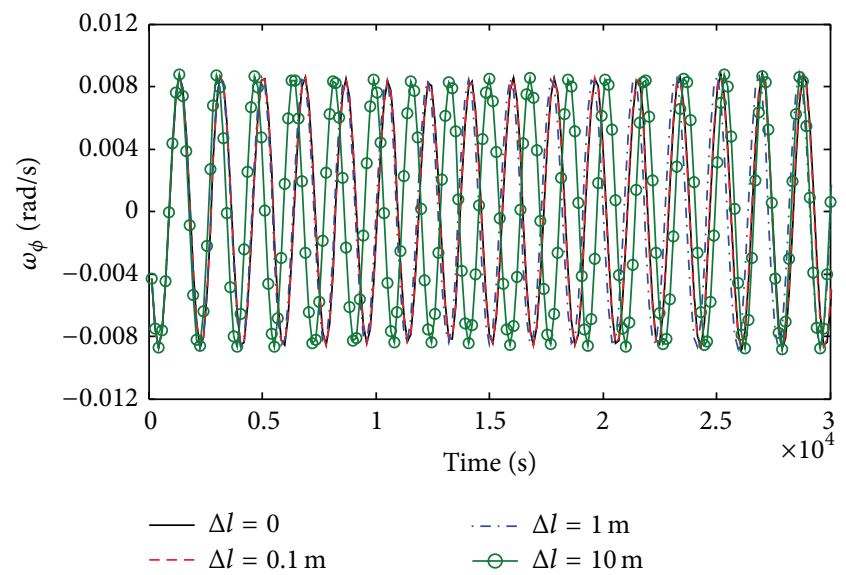

FIgURE 22: The time history of yaw angle $\phi$ with different $\Delta l, \tau=0$.

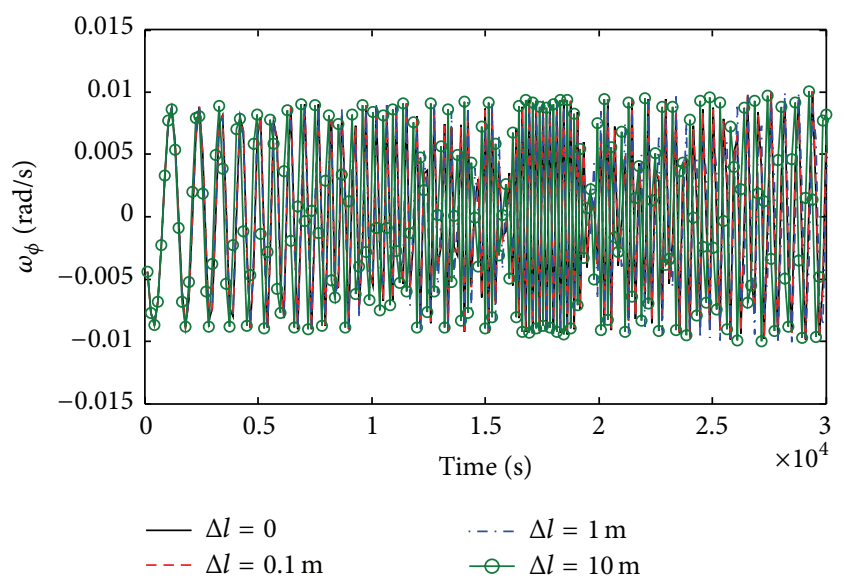

FIGURE 23: The time history of yaw angle $\phi$ with different $\Delta l, \tau=$ $250 \mathrm{kN} \cdot \mathrm{m}$.

Additionally, the structure bias of the MMET also contains the difference in payloads' mass $\Delta m$, which also changes the COM of the MMET and has the similar effect on 
the dynamics of the MMET compared to the effect of the difference in tethers' length on the dynamics of the MMET.
The transformation matrix from orbit coordinate to geocentric equatorial inertial coordinate is given by

$$
\mathbf{R}_{o \rightarrow I}=\left[\begin{array}{ccc}
\cos \Omega \cos f-\sin \Omega \cos i \sin f & -\cos \Omega \sin f-\sin \Omega \cos i \cos f & \sin \Omega \sin i \\
\sin \Omega \cos f+\cos \Omega \cos i \sin f & -\sin \Omega \sin f+\cos \Omega \cos i \cos f & -\cos \Omega \sin i \\
\sin i \sin f & \sin i \cos f & \cos i
\end{array}\right]
$$

where $f=\omega+\theta . \Omega, \omega$, and $i$ refer to the right ascension of ascending node, the argument of perigee, and orbit inclination, respectively.

Figure 24 shows the $3 \mathrm{D}$ orbit of the MMET in the geocentric equatorial inertial frame with the effect of the difference in tethers' length (the right ascension of ascending node $\Omega=90^{\circ}$, the argument of perigee $\omega=63^{\circ}$, and the orbital inclination $i=28.1^{\circ}$ ). It is shown that, after almost 11.4 circles, while the MMET is orbiting the Earth, $\Delta l$ has increasingly tiny influence on the orbit shape (especially the apogee) but no influence on the orientation of the orbital plane; the result coincides with the simulations in Section 6.

\section{Payload Injection Precision Analysis}

Figure 25 shows the schematic of a payload cislunar transfer at perigee; in this figure, $r_{a}, r_{p}$ refer to the perigee and apogee of the parking orbit of MMET, respectively. $v_{p}$ refers to the velocity of the chief satellite at perigee and $\Delta v$ refers to the required velocity increment of the payload from the parking orbit to the cislunar transfer orbit. While considering the tether length deviation $\Delta l$, the velocity $v_{p}$ is given by

$$
v_{p}=\sqrt{\frac{\mu}{r_{p}} \frac{2 r_{a}}{r_{a}+r_{p}}} .
$$

As analyzed above, the asymmetries of MMET have no influences on the perigee and just affect the apogee. If we set $\tilde{r}_{a}$ as the apogee of MMET's parking orbit with asymmetrical structure, then the velocity of the chief satellite at the perigee in this case is obtained by

$$
\widetilde{v}_{p}=\sqrt{\frac{\mu}{r_{p}} \frac{2 \widetilde{r}_{a}}{\widetilde{r}_{a}+r_{p}}} .
$$

Additionally, the perigee and apogee of the cislunar transfer orbit can be set as $r_{p}+l$ and $R_{m}$ (which is the radius of the moon around the Earth, assumed to be constant, and equals $3.844 \times 10^{8} \mathrm{~m}$ in this paper), respectively. Therefore, the velocity $v_{P_{1}}$ of the payload at the perigee required to be tossed into the cislunar transfer orbit is given by

$$
v_{P_{1}}=\sqrt{\frac{\mu}{r_{p}+l} \frac{2 R_{m}}{R_{m}+r_{p}+l}} .
$$

Combining (27) and (28), the required angular velocity $\dot{\psi}$ of the payload is obtained by

$$
\dot{\tilde{\psi}}=\frac{1}{l}\left(\sqrt{\frac{\mu}{r_{p}+l} \frac{2 R_{m}}{R_{m}+r_{p}+l}}-\sqrt{\frac{\mu}{r_{p}} \frac{2 \widetilde{r}_{a}}{\widetilde{r}_{a}+r_{p}}}\right) .
$$

It is shown that the asymmetries of the MMET will affect the apogee of the parking orbit; the result is that the velocity of the MMET at perigee will be affected correspondingly. Table 4 shows the relations between the velocity of the MMET at perigee and required angular velocity of the propulsion tether while considering the structural asymmetries. Furthermore, from (29) we can modify the rotational angular velocity to ensure that the payload can be tossed into the cislunar transfer orbit accurately.

\section{Conclusions}

In this paper, by using the Lagrange methods, the asymmetrical dynamic equations of the MMET caused by the tether length deviation and payload mass deviation have been presented. After that, the numerical simulations and theoretical analysis were presented. Some useful results are listed as follows:

(1) The attitude and orbit coupling analysis results illustrated that the external centrifugal force $\mathbf{F}_{C}$ caused by the structure asymmetries has linear relation with the tether length deviation $\Delta l$ (as well as the payload mass deviation $\Delta m$ ). It is shown that the external torque $\tau$ changes $\mathbf{F}_{C}$ by affecting the rotational angular velocity of the propulsion tether indirectly.

(2) The numerical simulation results of the error dynamics of MMET show that the tether length deviation has influence on the orbit of the chief satellite. Specifically, the apogee of the parking orbit decreases slightly while $\Delta l$ increases; however, the perigee does not change. What is more, the simulations results also show that there is a critical point $O_{c}$ in the parking orbit while considering the structural asymmetries; while MMET is moving from the perigee to $O_{c}$, the radius error $\Delta R$ is positive; reversely, while the MMET moves from $O_{c}$ back to the perigee, $\Delta R$ is negative. Additionally, the structural asymmetries have effects on the attitude elements (including the pitch angle $\psi$ and yaw angle $\phi$ ) of the MMET; it is shown that increasing $\Delta l$ leads to the increment of the pitch angle error $\Delta \psi$ with minor wiggles; however, 
TABLE 4: The relative orbital errors with difference in tethers' length.

\begin{tabular}{lcccc}
\hline Structure bias & $\widetilde{r}_{a}\left(\times 10^{6} \mathrm{~m}\right)$ & $\widetilde{v}_{p}\left(\times 10^{3} \mathrm{~m} / \mathrm{s}\right)$ & $v_{\mathrm{P}_{1}}\left(\times 10^{3} \mathrm{~m} / \mathrm{s}\right)$ & 10.7855435 \\
\hline$\Delta l=0$ & 10.360000 & 8.4775844 & 10.7855435 & 0.2307959 \\
$\Delta l=0.1 \mathrm{~m}$ & 10.359989 & 8.4775827 & 10.7855435 & 0.2307960 \\
$\Delta l=1 \mathrm{~m}$ & 10.359892 & 8.4775670 & 10.7855435 & 0.2307977 \\
$\Delta l=10 \mathrm{~m}$ & 10.358919 & 8.4774103 & 0.2308133 \\
\hline
\end{tabular}

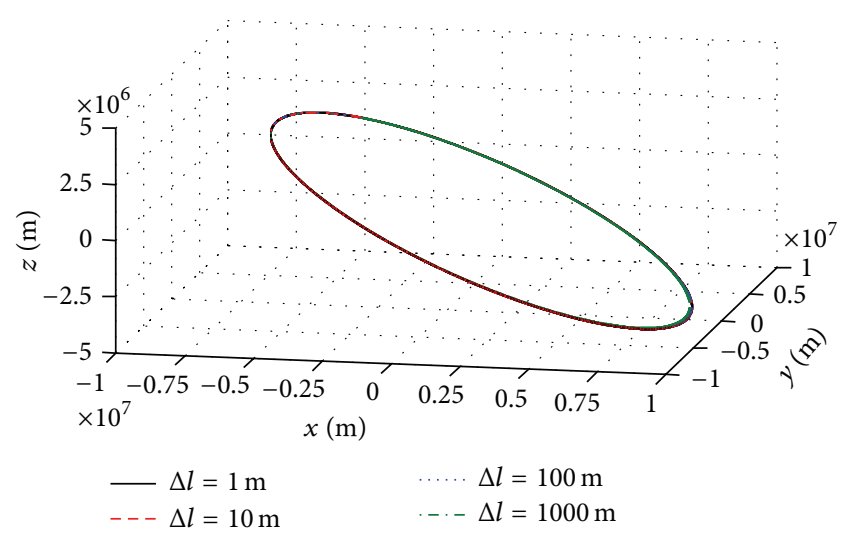

FIGURE 24: The orbit of MMET in geocentric equatorial inertial frame.

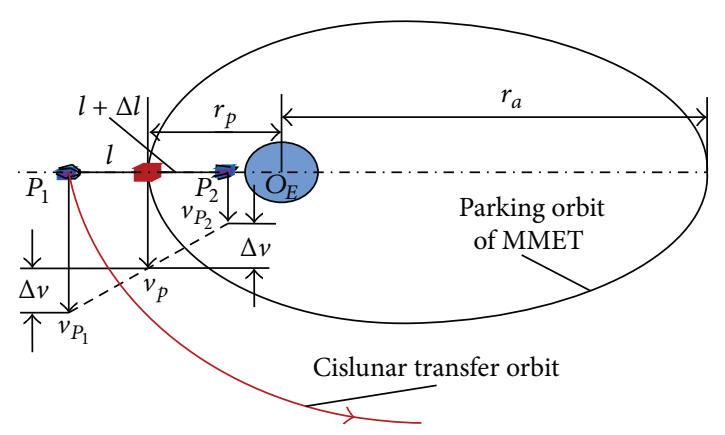

FIGURE 25: Schematic of payloads cislunar transfer at perigee.

the effects could be weakened by the external torque $\tau$. Furthermore, the pitch angular velocity $\dot{\psi}$ has been affected by the structural asymmetries and gravity gradient torque $\tau_{G}$ integratedly. It is shown that while increasing $\Delta l$, the maximum value of $\dot{\psi}$ increases slightly. What is more, $\dot{\psi}$ always reaches its maximum value at perigee because of the minimum effect of the gravity gradient torque $\tau_{G}$.

(3) The payload injection precision analyses by considering the structural asymmetries are presented lastly; it is illustrated that the orbit error caused by $\Delta l$ affects the velocity of the chief satellite at perigee nonlinearly; the result is that the required angular velocity $\dot{\psi}$ also needs to be modified to ensure that the payload can be tossed into the cislunar transfer trajectory accurately.

\section{Conflict of Interests}

The authors declare that there is no conflict of interests regarding the publication of this paper.

\section{Acknowledgments}

This work was supported by China Academy of Space Technology Innovation Foundation Project (CAST20090801) and was supported Zhejiang Provincial Natural Science Foundation (Y14F030005) and Zhejiang Provincial Science and Technology Project (2015C31160).

\section{References}

[1] A. C. Joseph, "Guidebook for analysis of tether applications," Final Report on Contract RH4-394049, 1985.

[2] J. A. Kornuta and S. M. Guo, "Momentum exchange tether as a hypersonic parachute during reentry for human missions," Journal of Spacecraft and Rockets, vol. 47, no. 4, pp. 571-579, 2010.

[3] L. Johnson, B. Gilchrist, R. D. Estes, and E. Lorenzini, "Overview of future NASA tether applications," Advances in Space Research, vol. 24, no. 8, pp. 1055-1063, 1999.

[4] M. P. Cartmell and D. J. McKenzie, "A review of space tether research," Progress in Aerospace Sciences, vol. 44, no. 1, pp. 1-21, 2008.

[5] P. H. Robert and U. Chauncey, "Cislunar tether transport system," Journal of Spacecraft and Rockets, vol. 37, no. 2, pp. 177186, 2000.

[6] P. H. Robert, "Commercial development of a tether transport system," in Proceedings of the 36th AIAA/ASME/SAE/ASEE Joint Propulsion Conference \& Exhibit, Las Vegas, Nev, USA, July 2000.

[7] P. H. Robert, "The cislunar tether transport system architecture," in Proceedings of the 2nd Lunar Development Conference, Las Vegas, Nev, USA, July 2000.

[8] M. P. Cartmell, "Generating velocity increments by means of a spinning motorised tether," in Proceedings of the 34th AIAA/ASME/SAE/ASEE Joint Propulsion Conference and Exhibit, pp. 1-12, AIAA, Cleveland, Ohio, USA, July 1998.

[9] S. W. Ziegler and M. P. Cartmell, "Using motorized tethers for payload orbital transfer," Journal of Spacecraft and Rockets, vol. 38, no. 6, pp. 904-913, 2001.

[10] R. P. Hoyt and C. Uphoff, "Cislunar tether transport system," Journal of Spacecraft and Rockets, vol. 37, no. 2, pp. 177-186, 2000.

[11] Y. Chen and M. P. Cartmell, "Dynamical modelling of the motorized momentum exchange tether incorporating axial elastic effects," in Advanced Problems in Mechanics, pp. 1-13, Russian Academy of Sciences, Saint Petersburg, Russia, 2007. 
[12] Y. Chen and M. P. Cartmell, "Hybrid fuzzy sliding mode control for motorised space tether spin-up when coupled with axial and torsional oscillation," Astrophysics and Space Science, vol. 326, no. 1, pp. 105-118, 2010.

[13] C. Murray, Continuous earth-moon payload exchange using motorized tethers with associated dynamics [Ph.D. thesis], University of Glasgow, Glasgow, UK, 2011.

[14] C. Murray and M. P. Cartmell, "Moon-tracking orbits using motorized tethers for continuous earth-moon payload exchanges," Journal of Guidance, Control, and Dynamics, vol. 36, no. 2, pp. 567-576, 2013.

[15] Y. Yang, N. M. Qi, Y. F. Liu, Z. Y. Xu, and M. Ma, "Influence of tapered tether on cislunar payload transmission system and energy analysis," Aerospace Science and Technology, vol. 46, pp. 210-220, 2015.

[16] N. M. Qi, Y. Yang, P. X. Huang et al., "Two dimensional dynamics of continuous cislunar payload transfer system considering structural deviation effect," Journal of Beijing University of Aeronautics and Astronautics, vol. 41, no. 11, pp. 2000-2009, 2015 (Chinese).

[17] S. W. Ziegler, The rigid body dynamic of tethers in space [Ph.D. thesis], University of Glasgow, 2003.

[18] N. A. Ismail, The dynamics of a flexible motorised momentum exchange tether (MMET) [Ph.D. thesis], University of Glasgow, Glasgow, UK, 2012.

[19] D. Liu and J. Zhao, Aero Spacecraft Dynamics, Harbin Institute of Technology Press, Harbin, China, 2003 (Chinese).

[20] J. Zhao, Orbital Dynamics of Spacecraft, Harbin Institute of Technology Press, Harbin, China, 2011 (Chinese).

[21] J. Missel and D. Mortari, "Removing space debris through sequential captures and ejections," Journal of Guidance, Control, and Dynamics, vol. 36, no. 3, pp. 743-752, 2013. 

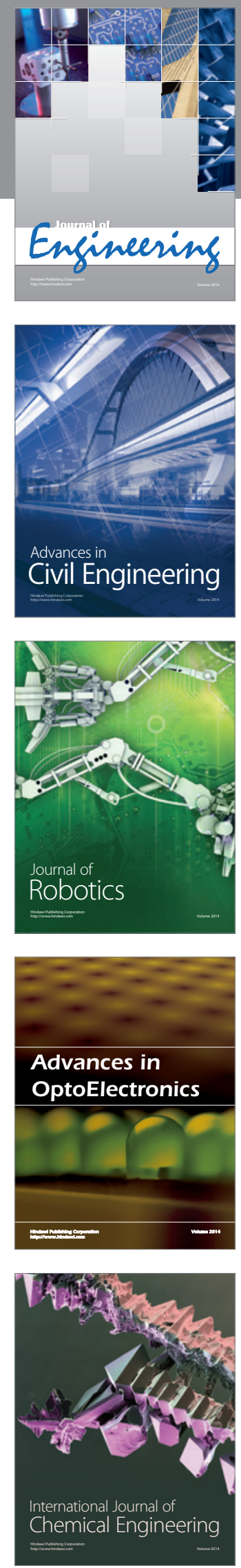

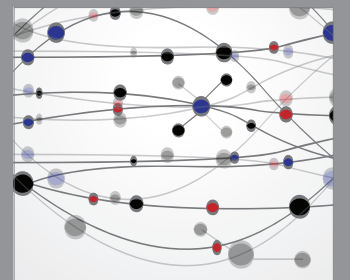

The Scientific World Journal
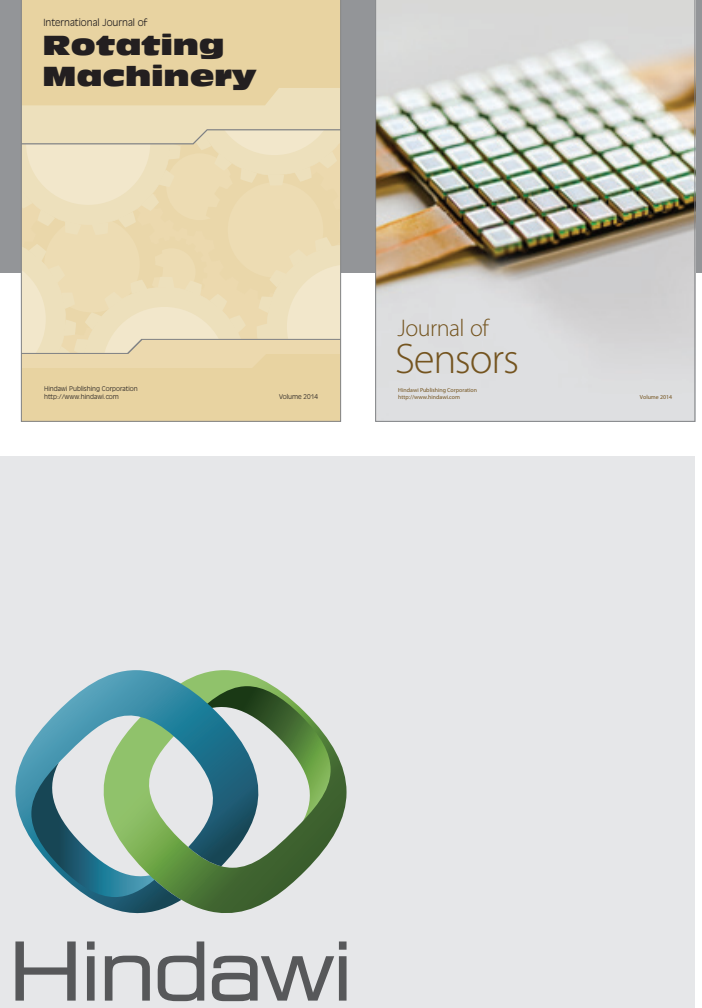

Submit your manuscripts at http://www.hindawi.com
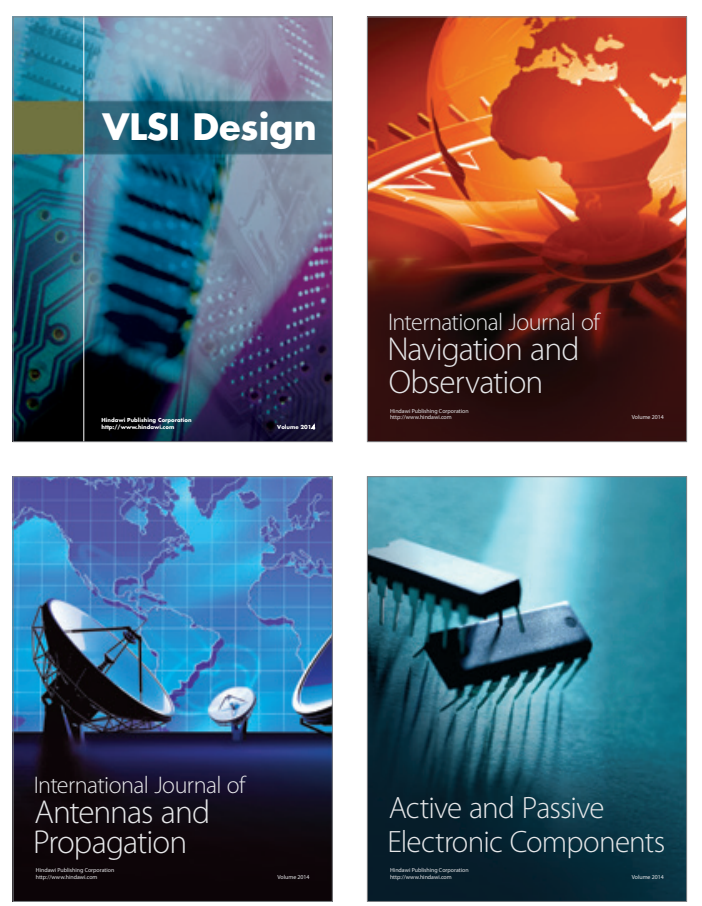
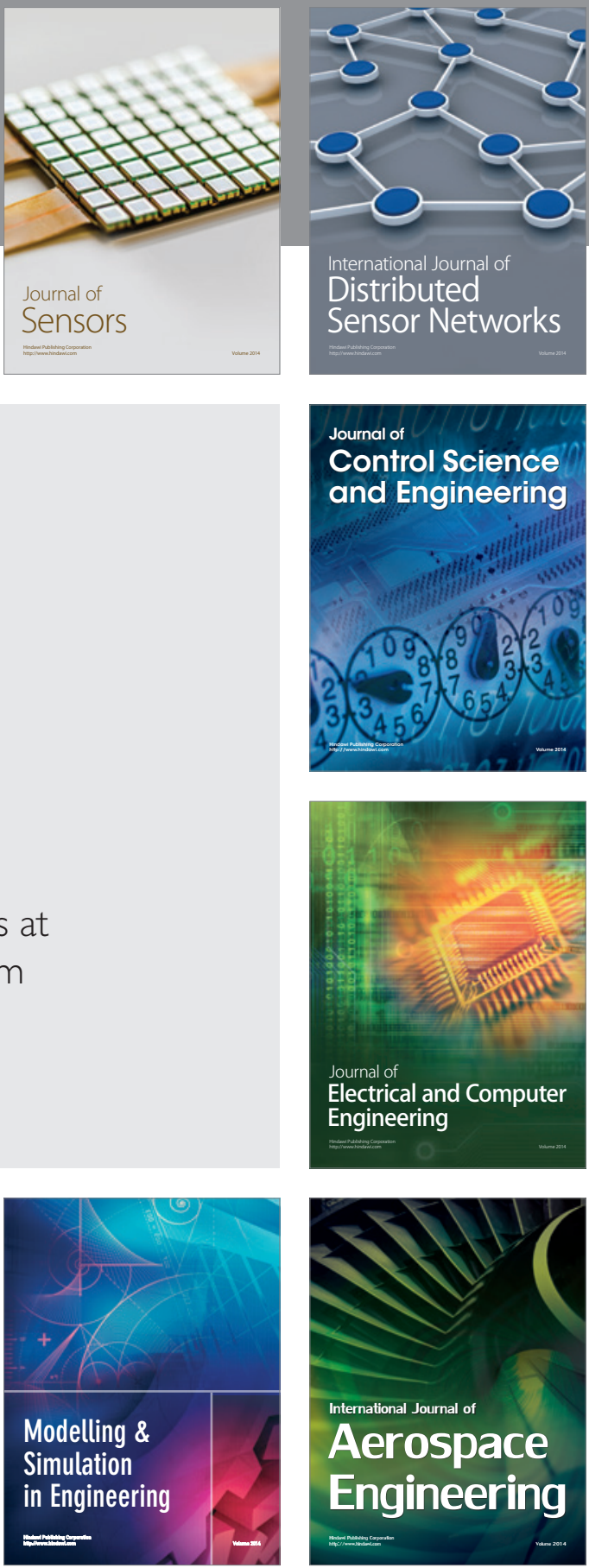

Journal of

Control Science

and Engineering
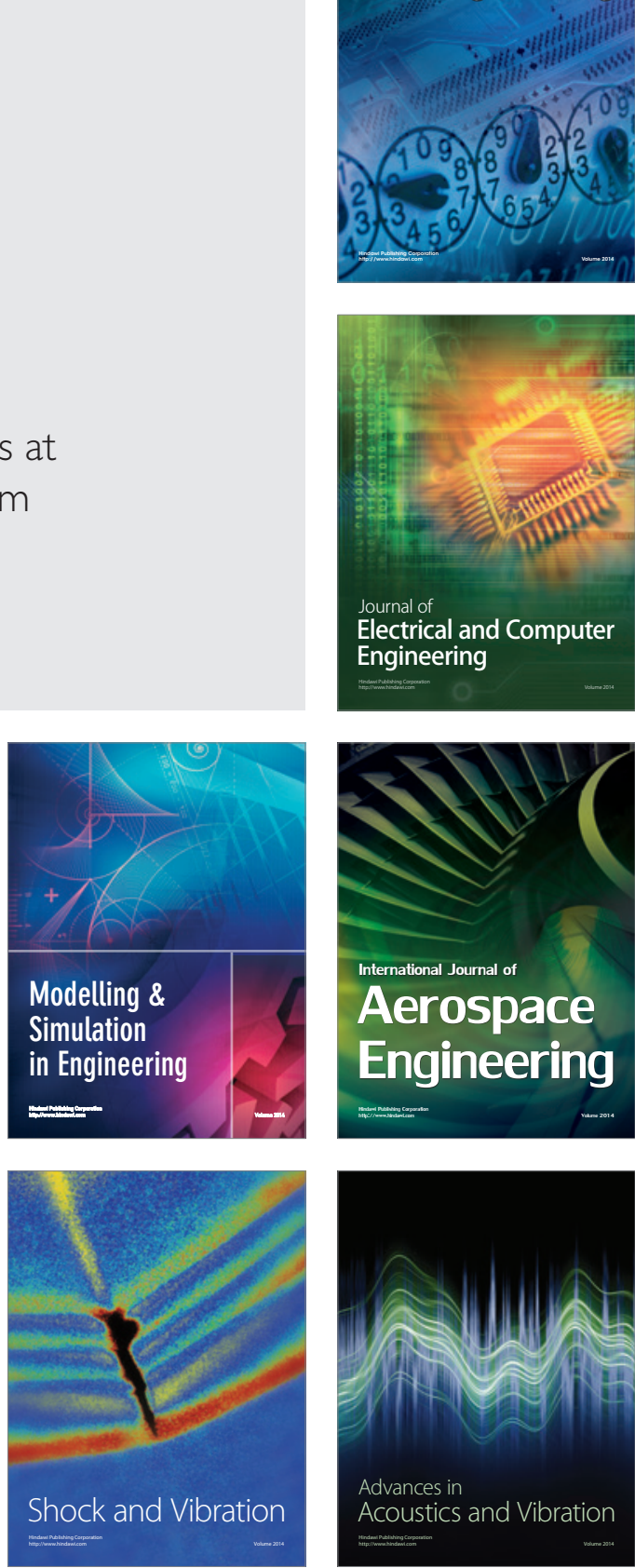